\title{
A putative viral defence mechanism in archaeal cells
}

\author{
REIDUN K. LILLEST $\varnothing L,{ }^{1}$ PETER REDDER, ${ }^{1}$ ROGER A. GARRETT ${ }^{1}$ and KIM BRÜGGER ${ }^{1,2}$ \\ ${ }^{1}$ Institute of Molecular Biology, University of Copenhagen, Sølvgade 83H, DK1307 Copenhagen K, Denmark \\ ${ }^{2}$ Corresponding author (brugger@mermaid.molbio.ku.dk)
}

Received April 27, 2006; accepted June 26, 2006; published online July 10, 2006

\begin{abstract}
Summary Clusters of regularly spaced direct repeats, separated by unconserved spacer sequences, are ubiquitous in archaeal chromosomes and occur in some plasmids. Some clusters constitute around $1 \%$ of chromosomal DNA. Similarly structured clusters, generally smaller, also occur in some bacterial chromosomes. Although early studies implicated these clusters in segregation/partition functions, recent evidence suggests that the spacer sequences derive from extrachromosomal elements, and, primarily, viruses. This has led to the proposal that the clusters provide a defence against viral propagation in cells, and that both the mode of inhibition of viral propagation and the mechanism of adding spacer-repeat units to clusters, are dependent on RNAs transcribed from the clusters. Moreover, the putative inhibitory apparatus (piRNA-based) may be evolutionarily related to the interference RNA systems (siRNA and miRNA), which are common in eukarya. Here, we analyze all the current data on archaeal repeat clusters and provide some new insights into their diverse structures, transcriptional properties and mode of structural development. The results are consistent with larger cluster transcripts being processed at the centers of the repeat sequences and being further trimmed by exonucleases to yield a dominant, intracellular RNA species, which corresponds approximately to the size of a spacer. Furthermore, analysis of the extensive clusters of Sulfolobus solfataricus strains $\mathrm{P} 1$ and $\mathrm{P} 2 \mathrm{~B}$ provides support for the presence of a flanking sequence adjoining a cluster being a prerequisite for the incorporation of new spacer-repeat units, which occurs between the flanking sequence and the cluster. An archaeal database summarizing the data will be maintained at http://dac.molbio.ku.dk/dbs/SRSR/.
\end{abstract}

Keywords: archaeal genomes, piRNA, plasmids, SRSRCRISPR, viruses.

\section{Introduction}

Clusters of regularly spaced repeats were first detected in Escherichia coli (Ishino et al. 1987), and were later found in archaea, both in chromosomes of the Haloferax genus of the kingdom Euryarchaeota (Mojica et al. 1993, 1995) and in conjugative plasmids of the Sulfolobus genus of the kingdom Crenarchaeota (She et al. 1998, Greve et al. 2004). The repeat clusters consist of a number of identical repeats interspaced with generally unique spacer sequences and have been assigned various acronyms in the literature including TREP, DVR, LCTR, SPIDR, SRSR and CRISPR. With the rapid progress in whole genome sequencing, it is now clear that repeat clusters are ubiquitous in the sequenced archaeal chromosomes, except that of Halobacterium sp. NRC-1, and they are present in $55 \%$ of the sequenced bacterial chromosomes. Striking for the Archaea, is that the chromosomal clusters are often both multiple and very large, such that in some Sulfolobus species, they constitute $>1 \%$ of the genome (Kawarabayasi et al. 2001, She et al. 2001).

Results from early experiments involving the transformation of repeat cluster-containing plasmids into species of the Haloferax genus implicated the clusters in chromosomal segregation (Mojica et al. 1995). Consistent with this view, it was demonstrated that, for both Euryarchaeota and Crenarchaeota, clusters tend to be replicated at the end of the replication cycle prior to chromosomal segregation (Zivanovic et al. 2002, Lundgren et al. 2004). Moreover, circumstantial evidence from studies of Sulfolobus conjugative plasmids supports the idea that plasmids carrying repeat clusters are more stably maintained in host cells (Greve et al. 2004).

Early attention focused on the structure and function of the repeat sequences, which generally carry a low level of nonpalindromic dyad symmetry. For Sulfolobus, a protein was isolated that bound specifically to the repeat and induced a distortion at its center, suggesting that it might have a role in folding the repeat clusters (Peng et al. 2003).

More recently, evidence has been presented that some spacer sequences in archaeal and bacterial chromosomes correspond closely to sequences occurring in extrachromosomal elements (Bolotin et al. 2005, Mojica et al. 2005, Pourcel et al. 2005). For archaea, sequence similarities were found with fuselloviruses and rudiviruses, as well as with a conjugative plasmid of Sulfolobus, and many matches lie within annotated open reading frames (ORFs) (Mojica et al. 2005). Moreover, for bacteria, cluster spacers of diverse strains of Streptococcus thermophilus were shown to produce matches to different bacteriophage or plasmid sequences (Bolotin et al. 2005). Furthermore, for Yersinia strains, spacers of the three small repeat clusters yielded multiple sequence matches with a chromosomal region containing a defective lambdoid prophage (Pourcel et al. 2005). These results are all consistent with the 
hypothesis that the spacer DNA derives from intracellular extrachromosomal elements.

Furthermore, evidence from comparative studies of Mycobacterium tuberculosis and Yersinia strains suggests that the sizes of repeat clusters can change by addition or deletion of one or more repeat-spacer units. In Yersinia, new repeat-spacer units can be added at the end of clusters, where a flanking or leader sequence is located (van Embden et al. 2000, Jansen et al. 2002, Tang et al. 2002, Pourcel et al. 2005). The mechanism by which this occurs could involve the products of a superoperon of genes that were originally implicated in DNA repair primarily in archaea and thermophilic bacteria (Makarova et al. 2002). These genes, some of which were later labeled cas genes, were considered to be co-functional with the repeat clusters because they are located close to chromosomal repeat clusters and are absent from bacterial chromosomes lacking repeat clusters (Jansen et al. 2002, Bolotin et al. 2005, Haft et al. 2005).

These results led to a common hypothesis for both archaea and bacteria that the cluster spacers are relics of an earlier, intracellular presence of extrachromosomal elements. The incorporation of their DNA into the repeat clusters then provides immunity against subsequent cellular invasion and propagation by identical, or closely related, genetic elements (Bolotin et al. 2005, Mojica et al. 2005). The hypothesis is strongly underpinned, at least for the archaea Archaeoglobus fulgidus and Sulfolobus solfataricus P1, by the finding that repeat clusters produce transcripts from one DNA strand, which may target and inactivate either gene transcripts or genes of the invading genetic elements (Tang et al. 2002, 2005). Moreover, such a mechanism is consistent with the finding of double-strandspecific endoribonucleases in both euryarchaeal and crenarchaeal species, which could be involved in the degradation of double helical RNA regions generated by the annealing of antisense-RNA and mRNAs (Stolt and Zillig 1993, Ohtani et al. 2004). Such a process is also reinforced by the structural characterization of euryarchaeal argonaute proteins which, in eukaryotes, have been implicated in the processing of interference RNAs (Parker et al. 2004, Song et al. 2004).

Recently, Marakova et al. (2006) extended the abovementioned knowledge by reassigning the cluster-associated cas genes as primarily encoding an RNA/DNA regulatory-processing system related to the eukaryal interference RNA (siRNA and miRNA) systems, which they defined as a prokaryotic interference RNA (piRNA).

In this article we summarize what is currently known about the structure and function of the repeat clusters of archaeal genomes and include some new data relevant to their structural and functional properties.

\section{Materials and methods}

\section{Sequencing of clusters in S. solfataricus $P 1$}

Long range PCR products were obtained across the chromosomal cluster regions of strain P1, which differ in sequence from those of strain P2, using the Herculase II kit (Stratagene,
La Jolla, CA) according to the protocol, with $150 \mathrm{ng}$ genomic DNA in a $25 \mu \mathrm{l}$ reaction. Similar regions were amplified in 1-2 kb sections with Taq DNA polymerase (New England Biolabs, Ipswich, MA) and 2 ng genomic DNA in a $15 \mu \mathrm{l}$ reaction. The PCR products were purified using QIAquick PCR purification kit (Qiagen, Westburg, Germany). Sequencing was performed on an ABI Prism 310 Genetic Analyzer (Applied Biosystems, Foster City, CA), where each $10 \mu \mathrm{l}$ sequencing reaction consisted of $1.4 \mu \mathrm{l}$ purified PCR product, $1.6 \mathrm{pM}$ primer and $2 \mu \mathrm{l}$ Big-Dye Terminator v.1.1 Cycle Sequencing Kit (Applied Biosystems). The reaction was run on a TRIOthermoblock (Biometra, Goettingen, Germany) $\left(30 \mathrm{~s}\right.$ at $96^{\circ} \mathrm{C}$, $15 \mathrm{~s}$ at $50{ }^{\circ} \mathrm{C}, 4 \mathrm{~min}$ at $\left.60{ }^{\circ} \mathrm{C}\right) \times 25$ and then maintained at $4{ }^{\circ} \mathrm{C}$, whereupon it was ethanol-precipitated and redissolved in $12.5 \mu \mathrm{l}$ Template Suppressing Reagent (Applied Biosystems). The sequences were analyzed with Sequencher (Gene Codes, Ann Arbor, MI), BLAST searches were performed against the Sulfolobus Database (http://dac.molbio.ku.dk/dbs/Sulfolobus/cbin/mutagen.pl).

\section{Preparation of total RNA and Northern blotting}

Sulfolobus acidocaldarius cells were grown at $78{ }^{\circ} \mathrm{C}$ in complex medium containing 2\% tryptone (Schleper et al. 1995). Total RNA was prepared from exponentially growing and stationary phase cells by the phenol-guanidium-thiocyanatechloroform method (Sambrook and Russell 2001) with DNase I treatment. Twenty $\mu \mathrm{g}$ of total RNA was fractionated in an $8 \%$ polyacrylamide gel with $7 \mathrm{M}$ urea, $90 \mathrm{mM}$ Tris, $64.6 \mathrm{mM}$ boric acid, 2.5 mM EDTA, pH 8.3, together with a 10-100 nt RNA ladder (Decade Marker System, Ambion, Huntingdon, U.K.) or a 0.1-2.0 kb RNA ladder (Invitrogen, Paisley, U.K.). The RNA was transferred onto nylon membranes (Hybond $\mathrm{N}^{+}$, Amersham Biosciences, Amersham, U.K.) using the Bio-Rad semi-dry blotting apparatus (Trans-blot SD, Bio-Rad, Hercules, CA). After immobilizing the RNAs using a Crosslinker (Stratagene), the nylon membranes were prehybridized for $1 \mathrm{~h}$ in $6 \times \operatorname{SSPE}\left(0.9 \mathrm{M} \mathrm{NaCl}, 60 \mathrm{mM} \mathrm{NaH} \mathrm{PO}_{4}, 4.6 \mathrm{mM}\right.$ EDTA and $\mathrm{pH} 7.4), 0.5 \%$ SDS and $5 \times$ Denhardt's solution at $59{ }^{\circ} \mathrm{C}$. Oligonucleotide primers (26-mers), complementary to each strand of a terminal spacer in saci-4 (5'-GATACGTTGCAGGCAGATGATGAGAG-3', 5'-CTCTCATCATCTGCCTGCAACGTATC- $3^{\prime}$ ), were end-labeled with $\left[{ }^{32} \mathrm{P}\right]$ ATP and T4 polynucleotide kinase. Hybridization was carried out at $59{ }^{\circ} \mathrm{C}$ in $6 \times$ SSPE, $0.5 \%$ SDS, $3 \times$ Denhardt's solution and $100 \mu \mathrm{g}$ $\mathrm{ml}^{-1}$ tRNA for $18 \mathrm{~h}$. The sample was washed three times at room temperature in $6 \times \mathrm{SSPE}$ and $0.1 \%$ SDS for $15 \mathrm{~min}$ each and subsequently at $59^{\circ} \mathrm{C}$ in the same buffer. Membranes were exposed to Ultra UV-G X-ray film (Dupharma, Kastrup, Denmark) for 3 days.

\section{Genome sequence analyses}

Genome sequences were downloaded from National Center for Biotechnology Information (NCBI), except that of Haloferax volcanii, which was obtained from The Institute for Genomic Research (http://www.tigr.org), and the Hyperthermus butylicus sequence (H.-P. Klenk, eGene Biotechnologie, 
Feldafing, Germany, unpublished data). All sequences were searched using the program LUNA obtainable from http://dac.molbio.ku.dk/bioinformatics/luna/. Short perfect repeats were identified with LUNA and the sequences were then extracted with a perl-script (available on request from this web-site). All clusters were further analyzed by BLAST searches against the Genbank databases. Matches to extrachromosomal elements were considered to be significant if they contained $>20$ identical nucleotides. If the matches were 25-40 bp, a few mismatches were allowed. A cut-off at $20 \mathrm{bp}$ was selected because a shorter sequence would be expected to occur randomly once in a $1.1 \mathrm{Mbp}$ genome.

\section{Results and discussion}

The archaea for which genome sequences are available fall into the major kingdoms Crenarchaeota and Euryarchaeota, and cover a wide range of optimal growth conditions. The crenarchaea are all extreme- or hyper-thermophiles, whereas the euryarchaea, including haloarchaea, methanoarchaea and thermophiles, grow optimally over a wide range of temperatures. The full names of the organisms, their optimal growth temperatures and the Accession numbers of their genome sequences are listed in Table 1.

The numbers of clusters and repeat-spacer units that occur in each archaeal chromosome or plasmid are presented in Table 1. Most chromosomes contain two to eight clusters, the exception being $M$. jannaschii, which contains 20. Furthermore, the chromosomes carry a large number of repeat-spacer units extending from 25 for $N$. pharaonis to 462 for $S$. tokodaii. On average, the thermophilic organisms carry more repeat-spacer units, but this is not a strict rule (Table 1).

Clusters also occur in extrachromosomal elements (Tables 1 and 2). Both conjugative plasmids of Sulfolobus, pNOB8 and pKEF9, exhibit a single cluster with six repeat-spacer units (She et al. 1998, Greve et al. 2004). Moreover, two plasmids of H. marismortui, pNG300 and pNG400, and two plasmids of $N$. pharaonis contain single clusters (Baliga et al. 2004, Falb et

Table 1. Summary of properties of the archaeal chromosomal and plasmid clusters.

\begin{tabular}{|c|c|c|c|c|c|}
\hline Organism/plasmid & Strain & $\begin{array}{l}\text { Optimal growth } \\
\text { temp. }\left({ }^{\circ} \mathrm{C}\right)\end{array}$ & $\begin{array}{l}\text { No. of } \\
\text { clusters }\end{array}$ & $\begin{array}{l}\text { Total no. } \\
\text { of repeats }\end{array}$ & $\begin{array}{l}\text { Accession } \\
\text { no. }\end{array}$ \\
\hline \multicolumn{6}{|l|}{ Crenarchaea } \\
\hline Hyperthermus butylicus & & $95-106$ & 2 & 93 & - \\
\hline Pyrobaculum aerophilum & IM2 & 100 & 5 & 136 & AE009441 \\
\hline Aeropyrum pernix & $\mathrm{K} 1$ & 95 & 4 & 89 & BA000002 \\
\hline Sulfolobus solfataricus & $\mathrm{P} 2 \mathrm{~B}$ & 80 & 7 & 425 & AE006641 \\
\hline Sulfolobus tokodaii & 7 & 80 & 6 & 462 & BA000023 \\
\hline Sulfolobus acidocaldarius & DSM639 & 75 & 5 & 227 & СР000077 \\
\hline plasmid pNOB8 & & 80 & 1 & 6 & AJ010405 \\
\hline plasmid pKEF9 & & 80 & 1 & 6 & AJ748321 \\
\hline \multicolumn{6}{|l|}{ Nanoarchaea } \\
\hline Nanoarchaeum equitans & Kin4-M & 100 & 2 & 42 & AE017199 \\
\hline \multicolumn{6}{|l|}{ Euryarchaea } \\
\hline Pyrococcus abyssi & GE5 & 103 & 5 & 62 & AL096836 \\
\hline Pyrococcus furiosus & DSM3638 & 100 & 8 & 208 & AE009950 \\
\hline Pyrococcus horikoshii & OT3 & 98 & 7 & 153 & BA000001 \\
\hline Thermococcus kodakaraensis & KOD1 & 85 & 3 & 77 & AP006878 \\
\hline Picrophilus torridus & DSM9790 & 60 & 3 & 120 & AE017261 \\
\hline Archaeoglobus fulgidus & DSM4304 & 83 & 3 & 152 & AE000782 \\
\hline Thermoplasma acidophilum & DSM1728 & 59 & 2 & 48 & AL139299 \\
\hline Thermoplasma volcanium & GSS1 & 60 & 3 & 36 & BA000011 \\
\hline Methanopyrus kandleri & AV19 & 98 & 5 & 41 & AE009439 \\
\hline Methanocaldococcus jannaschii & DSM2661 & 85 & 20 & 200 & L77117 \\
\hline Methanothermobacter thermoautotrophicus & $\Delta \mathrm{H}$ & 65 & 2 & 171 & AE000666 \\
\hline Methanosarcina barkeri & fusaro & 37 & 6 & 101 & $\begin{array}{l}\text { CP000099/ } \\
\text { CP000098 }\end{array}$ \\
\hline Methanosarcina acetivorans & $\mathrm{C} 2 \mathrm{~A}$ & 37 & 8 & 79 & AE010299 \\
\hline Methanosarcina mazei & Go1 & 37 & 8 & 136 & AE008384 \\
\hline Methanospirillum hungatei & JF-1 & 37 & 6 & 266 & СP000254 \\
\hline Methanosphaera stadtmanae & DSM3091 & 37 & 2 & 119 & CP000102 \\
\hline Methanococcoides burtonii & DSM6242 & 23 & 2 & 87 & СР000300 \\
\hline $\begin{array}{l}\text { Haloarcula marismortui } \\
\text { plasmids pNG300, pNG400 }\end{array}$ & ATCC 43049 & 45 & 3 & 129 & $\begin{array}{l}\text { AY596297/ } \\
\text { AY596298 }\end{array}$ \\
\hline Haloferax volcanii & & 45 & 3 & 76 & - \\
\hline Natronomonas pharaonis & DSM2160 & $43-45$ & 4 & 25 & CR936257 \\
\hline
\end{tabular}


al. 2005). For the N. pharaonis plasmid, one cluster is identical to a chromosomal cluster except that the latter contains an additional repeat-spacer unit (Table 2).

\section{Properties of the sequence repeats}

Repeat sequences vary in both length and sequence and are presented for each cluster in Table 2. The crenarchaeal genomes range in size from 24 to $26 \mathrm{bp}$, whereas the euryarchaea and the nanoarchaeon genomes vary from 26 to $37 \mathrm{bp}$. There is only limited conservation of many repeat sequences, with the left half showing the higher conservation, as was noted earlier (Peng et al. 2003). Nevertheless, some repeat sequences show major differences, especially in the right half of the sequence; compare, for example, the right halves of the repeats of plasmids pNOB8 and pKEF9 (Table 2). Most repeat sequences show some kind of weak, dyad symmetry generally in the form of interrupted and imperfect short inverted repeats and it has been shown, at least for the genus Sulfolobus, that this provides a recognition site for a repeat binding protein (Peng et al. 2003).

Within some clusters, the repeat sequence exhibits a little variation. For example, in the $S$. solfataricus cluster, ssol-95, the repeat sequence changes at the center of the cluster to another sequence (albeit with a single nucleotide change) (She et al. 2001). Therefore, we examined the constancy of repeat sequences within each genomic cluster and the results demonstrate that most clusters are not homogeneous in their repeat sequence (Table 2). Many carry one to four altered repeat sequences and two, the aforementioned ssol-95 cluster of $S$. solfataricus and stok-112 of S. tokodaii, show more dramatic changes. In addition, six of the 20 clusters in $M$. jannaschii carry two repeat sequences differing in their central regions (Table 2).

\section{Uniqueness of the spacer sequences?}

Spacers vary in size from 35 to $44 \mathrm{bp}$ between clusters as well as between organisms, and tend to be conserved within a cluster (Table 2). However, occasional exceptions were detected. In the $S$. solfataricus cluster ssol-91, a half spacer precedes two atypical repeat sequences, the second of which is followed by a regular repeat sequence and not a spacer. In $S$. tokodaii cluster stok-121, two atypical repeats are $18 \mathrm{bp}$ longer than the other repeats, but are followed by shorter spacers such that the repeat-spacer unit is conserved in size. In N. equitans, two spacers are $25 / 26$ bp longer than the others. The 56 bp spacers of $M$. kandleri are more than $10 \mathrm{bp}$ longer than those found in any other archaea.

All spacer sequences within a cluster and within a chromosome are generally different, but a systematic search revealed several exceptions. Spacers are occasionally repeated, sometimes more than once within a cluster, and can appear in different clusters within the same chromosome. The results demonstrate that 12 of the 28 chromosomes investigated carry one or more repeated spacers, which tend to be located in the larger clusters. The distributions of duplicated spacers are indicated in Table 3. There are no clear patterns for the arrangement of duplicated spacers, as some are arranged consecutively, others are located in different parts of a given cluster and a few occur in different clusters. The greatest number of duplicated spacers (36) occurs in the two clusters of M. thermautotrophicus (Table 3) and their distribution is illustrated for the larger mthe-124 cluster in Figure 1A. Although identical groups of spacer-repeat units have been observed in closely related strains, they have not been detected in different species.

\section{Integrity of the clusters}

Repeat clusters are generally highly conserved in their repeat sequences and in the sizes of their repeats and spacers. Such integrity extends to an almost complete lack of insertion sequences (ISs) or other mobile elements. This is surprising, given the large number of mobile elements in some archaeal genomes and the large variety of spacer sequences that could provide potential target sites for the insertion of mobile elements (Brügger et al. 2002). Nevertheless, one IS element (ISH4) was identified within the repeat cluster, hmar-57, of the H. marismortui megaplasmid, pNG400 (Table 2). Moreover, a minature inverted-repeat transposable element (MITE) occurs in the mace-33 cluster of $M$. acetivorans (Table 2), located at the center of a repeat sequence close to the end carrying the flanking sequence, but not in any of the other identical repeat sequences. The 132 bp MITE shares a 14 bp inverted terminal repeat and a 3 bp direct repeat with the IS element, ISMac11, which exists in the same chromosome and encodes a transposase likely responsible for the MITE transposition (Brügger et al. 2002).

\section{Properties of the flanking sequence}

Many chromosomal clusters carry a flanking sequence at one end, which is sometimes referred to as a "leader," although its function is unknown (see below). These sequences tend to be rich in short homopolynucleotide sequences and AT-rich regions, and they lack open reading frames (Jansen et al. 2002, Tang et al. 2002). Archaeal flanking sequences range in size from 132 bp for H. marismortui to 564 bp for M. kandleri (Table 2), and they directly adjoin the first repeat sequence of the cluster. Moreover, they invariably occur at the same end of the cluster, with respect to the strand orientation of the repeat sequence. There is an approximate direct correlation between the sequence length and the optimal growth temperature of the organism (Tables 1 and 2).

For chromosomes carrying multiple clusters with identical repeats, the flanking sequence (if present) is often conserved (Table 2). However, there is generally no conservation of the flanking sequence between organisms, with the exception of three Methanosarcina species, which share five highly similar $171 \mathrm{bp}$ sequences with $>87 \%$ sequence identity (Figure $2 \mathrm{~A}$ ).

Several clusters lack a flanking sequence, including those with different repeat sequences found in $P$. aerophilum and A. pernix (Table 2) and some with identical repeats found in S. solfataricus P2B (see ssol-7 and ssol-91 in Figure 1B). 
Table 2. Properties of the repeat clusters. Organisms are arranged in the same order as in Table 1. Symbols: $*=$ cluster containing an inserted transposable element; $\dagger=$ cluster located on a plasmid; and ${ }^{\#}=$ single repeats that lack a flanking sequence.

\begin{tabular}{|c|c|c|c|}
\hline $\begin{array}{l}\text { Organism/ } \\
\text { plasmid }\end{array}$ & Repeat sequence & $\begin{array}{l}\text { Repeats per cluster } \\
\text { (altered in sequence) }\end{array}$ & $\begin{array}{l}\text { Flanking } \\
\text { sequence } \\
\text { (conserved bp) }\end{array}$ \\
\hline H. butylicus & CTTGCAATTCTCTTTTGAGTTGTTC & $47(1), 46$ & $2(394)$ \\
\hline \multirow[t]{3}{*}{ P. aerophilum } & GTTTCAACTATCTTTTGATTTCTGG & $15(2), 18,14(2)$ & $3(257)$ \\
\hline & СТTTCAАTССТСТTTTTGAGATTC & 81 & \\
\hline & GTTTCAATTCTTTTGTAGATTCTTC & 8 & \\
\hline \multirow[t]{2}{*}{ A. pernix } & CTTGCAATTCTATCTCGAAGATTC & $1,27(8), 19(2)$ & $3(476)$ \\
\hline & СТTTCTATTCССTTTAGGGATATGC & 42 & \\
\hline \multirow[t]{3}{*}{ S. solfataricus } & СTTTCAATTCCTTTTGGGATTAATC & $1,103,95(47)$ & $3(502)$ \\
\hline & CTTTCAATTCTATAAGAGATTATC & $32,96(1)$ & $2(266)$ \\
\hline & CTTTCAATTCTATAGTAGATTAGC & $7,91(2)$ & \\
\hline \multirow[t]{3}{*}{ S. tokodaii } & СTTTCAATTCCTTTTGGGATTCATC & $74(2), 112(48)$ & $2(482)$ \\
\hline & CTTTCAATTCCATTAAGGATTATC & $48(1), 104(1), 121(4)$ & $3(269)$ \\
\hline & CTTTATTCATAATGCTAATTCCGT & 3 & \\
\hline \multirow[t]{2}{*}{ S. acidocaldarius } & GTTTTAGTTTCTTGTCGTTATTAC & 133(1), 78(5) & $2(239)$ \\
\hline & CTTTCAATCCCTTTTGGGATTCATC & $4(1), 11(1), 1$ & $3(506)$ \\
\hline pNOB8 & CTTTCAATTCTATAGTAGATTATC & $6 \dagger$ & \\
\hline pKEF9 & GTTGCAATTCCCTAAATGTGCGGG & $6 \dagger$ & \\
\hline N. equitans & СТTTCAАTATTTCTAATATATTAGAAAC & $13,29(1)$ & $2(190)$ \\
\hline \multirow[t]{2}{*}{ P. abyssi } & CTTTCAATTCTATTTTAGTCTTATTGGAAC & $23(3), 4(2), 27$ & \\
\hline & СТTTCCACACTACTAAGTTCTACGGAAAC & $7(2), 1$ & $2(401)$ \\
\hline P. furiosus & CTTTCAATTCTATTTTAGTCTTATTGGAAC & $\begin{array}{l}52(3), 21(1), 23(2), 31,46(2), 1^{\#}, \\
22,12(5)\end{array}$ & $7(524)$ \\
\hline \multirow[t]{2}{*}{ P. horikoshii } & CTTTCCACACTATTTAGTTCTACGGAAAC & $18(1), 25(3), 66(14), 1^{\#}$ & $3(526)$ \\
\hline & CTTTCAATTCTATTTTAGTCTTATTGGAAC & $18(1), 7(2), 18(1)$ & $2(258)$ \\
\hline T. kodakaraensis & CTTTCAATTCTCTTAGAGTCTTATTGCAAC & $16(7), 24(5), 33(7)$ & $3(437)$ \\
\hline \multirow[t]{2}{*}{ P. torridus } & CTTCCATACTATCTAGTAATTCTTAAAC & $15(1), 17(1)$ & $2(322)$ \\
\hline & CTTTCAATCCTATTTAGGTTATTATTTAAC & $88(2)$ & \\
\hline \multirow[t]{2}{*}{ A. fulgidus } & CTTTCAATCCCATTTTGGTCTGATTTCAAC & $60,48(1)$ & $2(347)$ \\
\hline & СТTTCAATCTCCATTTTCAGGAGCCTCССТTTCTTAC & $44(4)$ & \\
\hline T. acidophilum & CTTTCAATCCTATTAAGGTTCTATTTTAC & $47(1), 1^{\#}$ & \\
\hline T. volcanium & CTTCCATACTAACTAGTACATCTTAAAC & $19(1), 16(1), 1$ & $3(287)$ \\
\hline M. kandleri & GTTTCATTACCCGTATTATTACGGGTTAATTGCGAG & $12(2), 5,8(1), 4(2), 12(3)$ & $5(564)$ \\
\hline \multirow[t]{2}{*}{ M. jannaschii } & TTTCCATTCCGAAACGGTCTGATTTTAAT/ & $26(1), 3(1), 4(2), 1,16(2), 24(4)$ & $19(440)$ \\
\hline & TTTCCATCCTCCAAGAGGTCTGATTTTAAC & $\begin{array}{l}12(2), 15,7(2), 13(2), 2,3(1), 14(4) \\
14(7), 5(1), 10(1), 1,9(2), 9(3), 12(1)\end{array}$ & \\
\hline $\begin{array}{l}\text { M. thermoauto- } \\
\text { trophicus }\end{array}$ & ATTTCAATCCCATTTTGGTCTGATTTTAAC & 124,47 & $2(460)$ \\
\hline \multirow[t]{3}{*}{ M. barkeri } & GTTTCAATCCCTCTAAGGCCTGATTTTAAC & $51(2)$ & \\
\hline & GTTTCAATCCTTGTTTTAGTGGATCTTGCTCACGAAT & $1^{\#}, 4(2), 1^{\#}, 19(1)$ & $1(171)$ \\
\hline & GTTTCCATAACCGAAAGGTTGTGGCAGAATTGAAGC & $25(5)$ & \\
\hline \multirow[t]{2}{*}{ M. acetivorans } & GTTTCAATCCTTGTTTTAGTGGATCTTGCTCGCGAAT & $1^{\#}, 7,33(2)^{*}, 2,1^{\#}, 1^{\#}, 2(1)$ & $2(171)$ \\
\hline & GTTTCAATCCCTCTAAGGTCTGATTTTAAC & $31(1)$ & \\
\hline M. mazei & GTTTCAATCCTTGTTTTAGTGGATCTTGCTCACGAAT & $1^{\#}, 2(1), 2(1), 1^{\#}, 1^{\#}, 47(7), 1^{\#}, 81(8)$ & $2(171)$ \\
\hline \multirow{3}{*}{$\begin{array}{l}\text { Methanospirillum } \\
\text { hungatei }\end{array}$} & GTTGCAAGTGACCCGAAAATAGAAGGGTATGGCAAC & $31,8,37(2)$ & $2(210)$ \\
\hline & GTTTCAATCCCTATCGGGTTTTCTTTTCCATTGTGAC & $44(1), 66$ & \\
\hline & GGTTCATCCCCATACACACGGGGAACTC & 80 & \\
\hline \multirow{2}{*}{$\begin{array}{l}\text { Methanosphaera } \\
\text { stadtmanae }\end{array}$} & GTTTAAAATAGACTTAATAGTATGAAAAC & $62(2)$ & \\
\hline & СТTTCAАTTTCATTATGATCTTATTCTATT & 57 & \\
\hline \multirow{2}{*}{$\begin{array}{l}\text { Methanococcoides } \\
\quad \text { burtonii }\end{array}$} & GAGTTCCCCATGCATGTGGGGATAAACCG & 65 & \\
\hline & GTTTCAATCCCTCTAAGGTCTGATTTTAAC & $22(2)$ & \\
\hline \multirow[t]{2}{*}{ H. marismortui } & GCTTCAACCCCACAAGGGTCCGTCTGAAAC & $48(13) \dagger$ & \\
\hline & GCTTCAACCCCACGAGGGTCCGTCTGTAAC & $24(1) \dagger, 57(1)^{* \dagger}$ & $2(132)$ \\
\hline & CTTCAATCCCACAAGGGTTCGTCTGAAAC & $25(1), 11(1), 40$ & $2(169)$ \\
\hline \multirow[t]{2}{*}{ N. pharaonis } & GCTTCAACCCCACAAGGGTTCGTCTGAAAC & $5(1), 3(2) \dagger$ & \\
\hline & GTCGAGACGGACTGAAAACCCAGAACGGGATTGAAAC & $9(1), 8(1) \dagger$ & \\
\hline
\end{tabular}


Protein genes associated with clusters

So far, the only protein that has been shown to interact directly with a cluster is the genus-specific SSO0454 from S. solfataricus $\mathrm{P} 2$, which binds and distorts the repeat sequence (Peng et al. 2003). However, superoperons containing $>20$ genes often flank one or more repeat clusters within archaeal and bacterial chromosomes (Jansen et al. 2002). Because they are absent from bacterial genomes that lack repeat clusters, they were inferred to be co-functional with the repeat clusters (Jansen et al. 2002). Although some of these genes (now denoted cas or csa genes) were earlier annotated as unusual DNA repair enzymes (Makarova et al. 2002), they have recently been reassigned to the regulation and processing of the repeat clusters and to a putative role in piRNA function (Makarova et al. 2006). Predicted functions of the more common gene products are listed in Table 4. Some of the genes show a degree of specificity for different archaeal or bacterial phyla including five archaea-specific genes (denoted csal to csa5) (Bolotin et al. 2005, Haft et al. 2005), although we find csa2 homologs in some bacterial genomes. Cas 5 and cas 6 should not be confused with identically named genes exclusive to bacterial genomes lacking cas2, cas3 and cas4 (Bolotin et al. 2005). An overview of the genes commonly found in archaeal genomes is presented in Table 5.

Typical examples of fairly conserved gene orders present in superoperons of crenarchaea and euryarchaea are presented in Figure 3 for the most commonly occurring genes. All eury-
Table 3. Spacer sequences repeated either within a cluster or between different clusters of the same organism.

\begin{tabular}{|c|c|c|c|c|}
\hline $\begin{array}{l}\text { Organism/ } \\
\text { plasmid }\end{array}$ & $\begin{array}{l}\text { Repeated } \\
\text { once in } \\
\text { a cluster }\end{array}$ & $\begin{array}{l}\text { Repeated more } \\
\text { than once in } \\
\text { a cluster }\end{array}$ & $\begin{array}{l}\text { Present in } \\
\text { different } \\
\text { clusters }\end{array}$ & $\begin{array}{l}\text { Total no. } \\
\text { of repeat- } \\
\text { ed spacers }\end{array}$ \\
\hline H. butylicus & 2 & & & 4 \\
\hline S. solfataricus & 1 & & 3 & 8 \\
\hline S. tokodaii & 2 & 1 & 5 & 17 \\
\hline $\begin{array}{l}\text { S. acido- } \\
\text { caldarius }\end{array}$ & 2 & 1 & & 9 \\
\hline P. furiosus & 1 & & 3 & 8 \\
\hline P. torridus & 10 & & & 20 \\
\hline A. fulgidus & & & 1 & 2 \\
\hline M. jannaschii & 2 & 2 & & 14 \\
\hline $\begin{array}{l}\text { M. thermauto- } \\
\text { trophicus }\end{array}$ & 18 & & & 36 \\
\hline M. barkeri & 1 & & & 2 \\
\hline M. mazei & 2 & & & 4 \\
\hline $\begin{array}{l}\text { H. marismortui } \\
\text { pNG300 }\end{array}$ & 1 & & & 2 \\
\hline $\begin{array}{l}\text { H. marismortui } \\
\text { pNG400 }\end{array}$ & 2 & & & 4 \\
\hline
\end{tabular}

archaeal genomes lack Csal, except for A. fulgidus. Each gene generally occurs once per superoperon, although duplicate copies occur in $M$. jannaschii and T. kodakaraensis. A few genes, including cas3, cas5, cas6, csa2, csa3 and COG2254, are sometimes located distantly from the repeat clusters, most

\section{A}

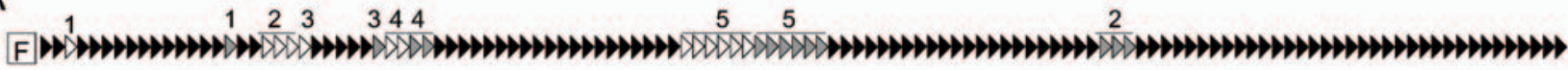

B

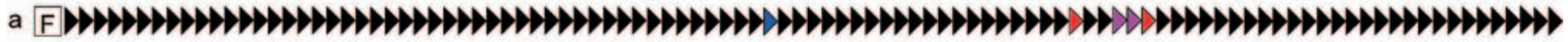

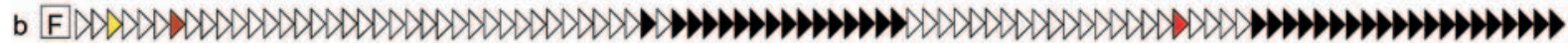
c FDD

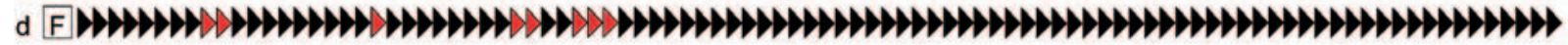
e DWD

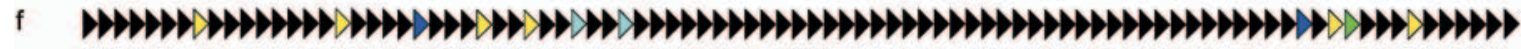

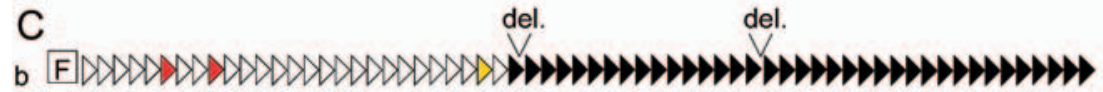

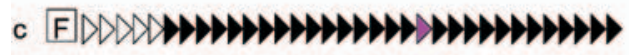
e MWW

Figure 1. (A) A map of mthe-124 from M. thermoautotrophicus showing the locations of duplicated spacer-repeat units, or groups of units, labeled 1-5. Each triangle represents a spacer-repeat unit. Duplicated spacer-repeat units are shaded in gray. (B) Schematic representation of the six clusters which occur in the genome of S. solfataricus P2B (She et al. 2001). Each triangle represents a spacer-repeat unit. The colored triangles are coded to indicate the archaeal viruses, plasmids or chromosomes (S. solfataricus or A. fulgidus) that yield good matches with the spacer sequence. (C) A corresponding scheme is presented for three of the six clusters (ssol-95, ssol-32 and ssol-7) that are present in the chromosome of the closely related strain S. solfataricus P1 (Accession numbers: DQ831675, DQ831676 and DQ831677). Open triangles denote those spacer-repeat units that differ in sequence between the strains P1 and P2A. Abbreviation: del. indicates the two sites where deletion of several spacer-repeat units has occurred in strain P1 (or where insertions have occurred in strain P2B). 
A

M. mazei SRSR-81

M. acetivorans SRSR-7

M. barkeri SRSR-19

M. mazei SRSR-47

M. acetivorans SRSR-33

M. mazei SRSR-81

M. acetivorans SRSR-7

M. barkeri SRSR-19

M. mazei SRSR-47

M. acetivorans SRSR-33

M. mazei SRSR-81

M. acetivorans SRSR-7

M. barkeri SRSR-19

M. mazei SRSR-47

M. acetivorans SRSR-33

\begin{abstract}
TATA-like

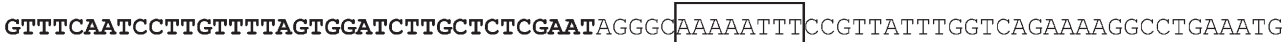
GTTTCAATCCTTGTTTTAGTGGATCTTGCTCTCGAATAGGGOAAAATTTT CCGTTATTTGGCCGGAAAAGGCCTGAAATC GTTTCAATCCTTGTTTTAGTGGATCTTGCTCACGAATAGGGDAAAAATTTCCGTTATTTGGCTAGAAAAGGCCTGAAATG GTTTCAATCCTTGTTTTAATGGATCTTGCTCGCGAATAGGGOAAAAATTT CCGTTATTTGGCCAGAAAAGGCCTGAAATG GTTTCAATCCTTGTTTAATGGATCTTGCTCGCGAATAGGGDAAAAATTTCCGTTATTACGCCAGAAAAGGCCTAAAATC
\end{abstract}

AGCCCTTTTTGGGGCTAAAATTCTTGCTGGAAAATTATCAAACCCTTTATAAATACAAGAAAAAAAGGGTTTTTTTAGT GCCCCTTTTTTGGGGTAAAATTCTTGCTGGAAAATTATCAAACCCTTTATAAATACAAGAAAAACAGGG---TTATAGT AGTCCTTTTATGGAGTTGAAATTCTTGTTGGAAAATTATCAAACCCTTTATAAATACAAGAAAAACAGGGT--TTCTAGC AGCCCTTTTTCAGGGCTAAAATTCTTGCTGGAAAATTATCAAACCCTTTATAAATACAAGAAAAAC-------TTCTCAT ACCTCTTTTTTGGACCTGAATTCTTGCTGGAAAATTATCAAACCCTTTATAAATACAAGAAAAAC-------TTCTTAA

GGTTTGGAAACATCTCAAAATATGCTTTTCTTTCTATTAAACTTTTGTTTTTAAATCTGT--GGAAG GGTATGGAGACATCTCAAAATATGCTTTTCTTTCTATTAAACTTTTGTTTTTGATTGCTACTGTTGT GGTTTGGAAACATTCTGATATATGCCTTTCTTTCTATTTAACTTTTGTATTTAAAAGCGTTCTAAGT A---CGCAGATATTCCGGTAACAGC------------AATCTACGCATTTT--------CTAGA

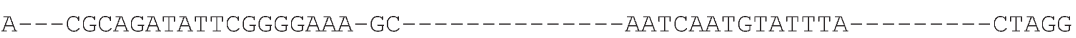

B

P. abysSi SRSR-23

P. abyssi SRSR-1

P. abysSi SRSR-23

P. abysSi SRSR-1

\begin{abstract}
СтTTCCACACTACTAAGTTCTACGGAAACCAGGAGAAAGTGCATGGGTTCATTGACGCAATCAGTTCAATCGACTTTCCA

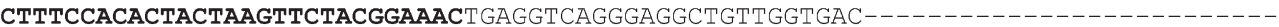
TATA-like

САCTACTAAGTTCTACGGAAACCCTTGGGATTTTTCCTTTAT-- 278 nt --AAGCCTTGAAAA ------AAGTTATACGGAAACCCTTGAAATTTTTGCTTGTT-- 278 nt --AAGCTTTGAGAA
\end{abstract}

Figure 2. (A) Alignment of the five conserved flanking sequences adjoining the first repeat (in bold type) of five clusters of the three Methanosarcina strains. A putative TATA-like box is outlined. (B) Alignment of two flanking regions of the P. abyssi genome where paby-1 appears to exhibit a defective start site.

commonly in the genomes of the three Pyrococcus species and A. fulgidus.

Organisms with multiple clusters carrying the same repeat sequence exhibit only a single superoperon adjacent to one cluster (Jansen et al. 2002). In some genomes, the superoperons physically link two, or even three, clusters with identical repeat sequences (e.g., in P. aerophilum, S. solfataricus, S. tokodaii and H. marismortui). In contrast, organisms containing two or more clusters with different repeat sequences generally have two or more superoperons (Jansen et al. 2002), except for P. aerophilum, A. pernix and S. tokodaii, which each have one. Exceptionally, superoperons are absent from the chromosomes of T. acidophilum and P. abyssi and plasmids pNOB8 and pKEF9, which all carry repeat clusters (Table 5).

In $M$. acetivorans and M. barkeri, a superoperon links clusters with dissimilar repeats and both the gene order (cas6cas4-cas1-cas2-Cluster1-csa2-cas5-cas3-Cluster2) and sequence ( $>94 \%$ identity) are conserved. Since the repeat sequences of the corresponding pairs of clusters are identical between these two organisms, this suggests that a lateral transfer event of both superoperon and clusters has occurred. However, such an event must have happened at an early stage of cluster development, because no similarities were detected between the spacer sequences of the two organisms.

Table 4. Predicted functions of common cas-genes in archaea. Data are summarized from Makarova et al. 2006 and earlier papers (Jansen et al. 2002, Makarova et al. 2002, Haft et al. 2005). Symbols: * $=$ formerly COG3578; and $* *=$ formerly COG3574.

\begin{tabular}{lll} 
Family/cas-name & Predicted function & Comments \\
\hline COG1518/cas1 & Nuclease/integrase & Possibly involved in inserting new DNA sequences \\
COG1343/cas2 & Nuclease & Possibly involved in inserting new DNA sequences \\
COG1203/cas3 & DNA helicase & Often fused to HD-nuclease domain/ related to COG2254 \\
COG2254 & HD-like nuclease & \\
COG1468/cas4 & RecB-like nuclease & \\
COG1688/cas5 & RNA-binding & "Ramp" superfamily \\
COG1583/cas6 & RNA-binding & "Ramp" superfamily \\
COG4343*/csa1 & RecB-like nuclease & \\
COG1857/csa2 & Nuclease & \\
COG2462/csa3 & HTH-type transcriptional regulator & \\
COG1353 & RNA polymerase & "Loosely" associated with repeat clusters \\
AF0070/csa4 & None & Crenarchaea-specific \\
AF1870**/csa5 & None & Crenarchaea-specific \\
\hline
\end{tabular}




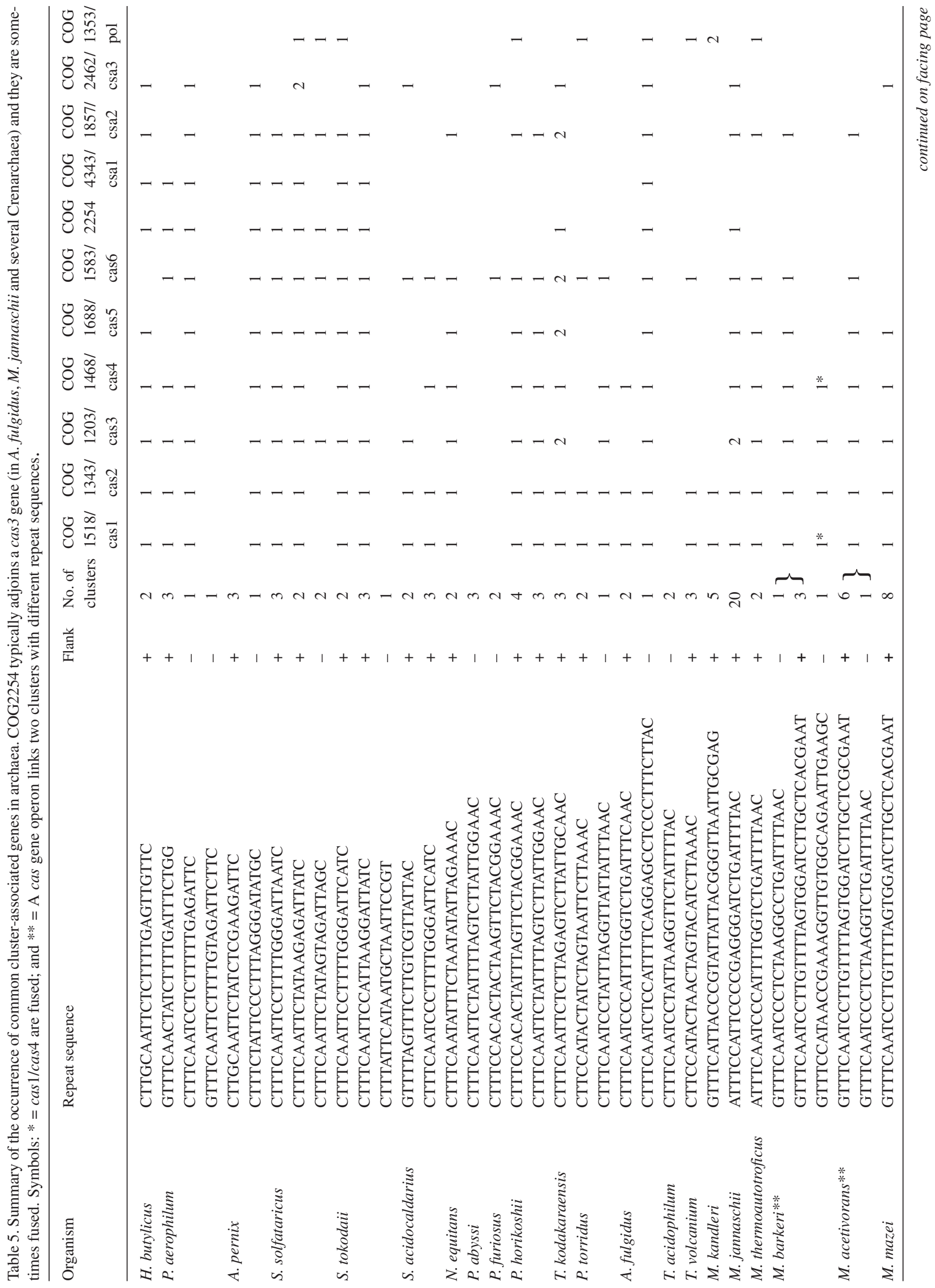




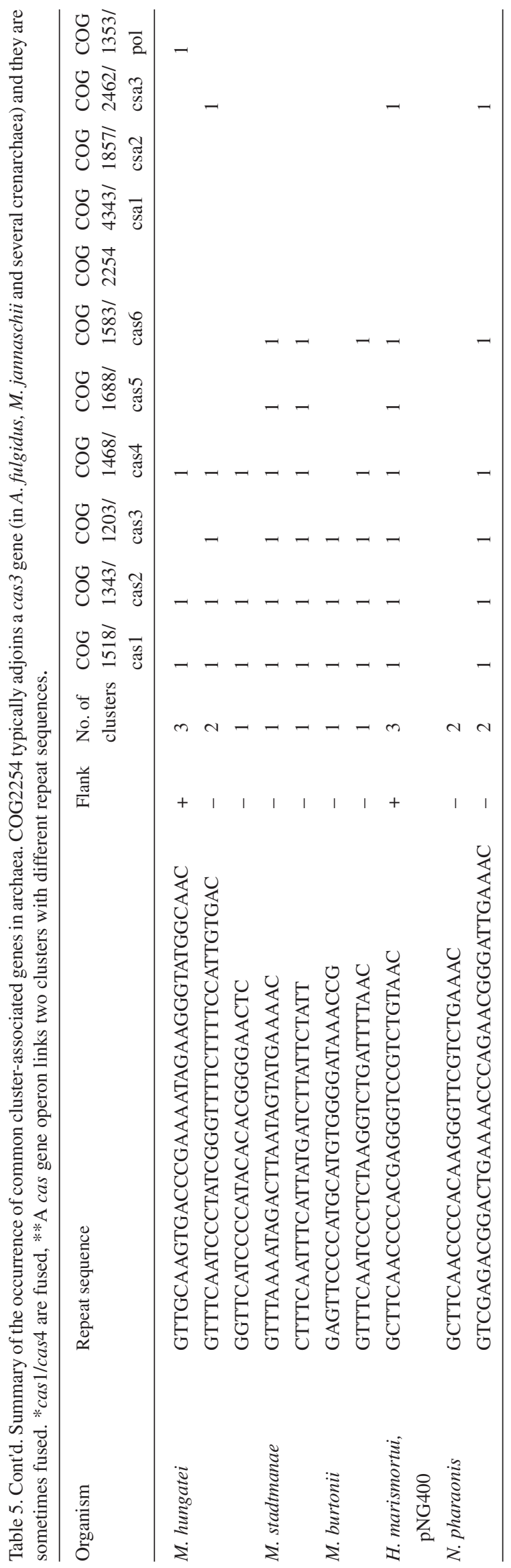

\section{Development of the clusters}

The only relevant study of cluster development was performed on related bacterial strains of $M$. tuberculosis (van Embden et al. 2000) and of Yersinia (Pourcel et al. 2005). Examination of 26 strains of $M$. tuberculosis revealed several differences in a large repeat cluster consistent with insertions/deletions of internal repeat-spacer units having occurred. For the related Yersinia strains, a similar phenomenon was observed, but it was also inferred that repeat-spacer units could have been added at the cluster end adjoining the conserved flanking sequence.

In order to shed some insight on how clusters may develop and change in archaea, we completely sequenced three of the six large cluster regions of $S$. solfataricus $\mathrm{P} 1$ and compared the spacer content with those of strain P2B. The two highly similar strains were originally sampled about one meter apart from a small stream at Pisciarelli, Naples (W. Zillig, deceased, personal communication). A comparative repeat-spacer alignment of the two strains (Figures $1 \mathrm{~B}$ and 1C) demonstrates the following: (1) new repeat-spacer units are added at, or near, the end of clusters $b$ and $c$, which both exhibit an adjoining flanking sequence; (2) the large $b$ clusters are more active in adding new repeat-spacer units than the smaller $c$ clusters; (3) no new repeat-spacer units are added to the $e$ clusters, which lack a flanking sequence, but the clusters remain highly conserved in sequence; (4) deletion and/or insertion of single, or multiple, repeat-spacer units can occur; and (5) deletion/insertion of repeat-spacer units occurs precisely, reinforcing the idea that the structural integrity of the clusters is important for their function.

Both data found in the literature (Pourcel et al. 2005) and the results presented in Figures $1 \mathrm{~B}$ and $1 \mathrm{C}$ are consistent with new spacer-repeat units being added exclusively adjacent to the flanking sequence. Moreover, since no new units were added to the repeat clusters lacking a flanking sequence (cluster ssol-32 in Figures $1 \mathrm{~B}$ and $1 \mathrm{C}$ ), this region is likely to provide a binding site for the cas genes involved in the copying-insertion events. Although there is no insight into the mechanism by which repeat-spacer units are added to a cluster, the process is likely to involve reverse transcription of mRNAs and recombination (Makarova et al. 2006).

There are several instances of a flanking sequence being followed by a single repeat or repeat-spacer unit (Table 2), consistent with the clusters developing from one end, but possibly also indicating a defective start site. One such region from $P$. abyssi is shown where the flanking sequence adjoins a one half repeat and truncated spacer, followed by a full repeat, and it is aligned with the start of paby-23 from the same genome (Figure 2B). There are also a few examples of single repeats that lack flanking sequences, mainly from the genera Pyrococcus and Methanosarcina (Table 2).

The preceding data suggest that development of clusters is primarily dependent on a combination of cas genes and the flanking sequence. An alternative hypothesis is that repeat clusters are spread intercellularly by plasmids (Godde and Bickerton 2006), and, at least for the Methanosarcina species, 
Crenarchaeota

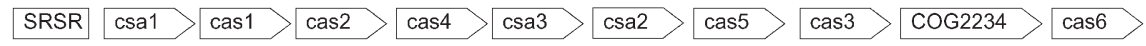

Euryarchaeota

SRSR $\operatorname{cas} 2<$ cas1 $<$ cas4 cas3 cas5 csa2 cas6 csa3 >
Figure 3. Typical composition and orientation of the major gene components of superoperons which are closely linked to repeat clusters in crenarchaea and euryarchaea. The cas genes and their COG identities are given in Tables 4 and 5. there is clear sequence evidence for the lateral transfer of a superoperon of cas genes and all its flanking sequences (Figure 2). Moreover, the presence of repeat clusters in conjugative plasmids provides a possible mechanism for intercellular transfer. However, the repeat cluster of pNOB8 does not appear in the $S$. tokodaii chromosome, where a closely similar plasmid sequence is encaptured (Kawarabayasi et al. 2001).

\section{The role of $R N A$}

Experimental studies have demonstrated that RNA is transcribed from the repeat-clusters of the euryarchaeon, A. fulgidus, and the crenarchaeon, S. solfataricus (Tang et al. 2002, 2005). Examination of clone libraries of reverse transcripts prepared from total cellular RNA $(<500 \mathrm{nt})$ yielded sequences of a series of cluster-encoded small RNAs (22 from A. fulgidus and one from Sulfolobus). Since the 5'-terminal sequence of each RNA was lacking (by about 10-25 nt), the start position could only be assigned approximately within a repeat sequence, while the $3^{\prime}$-terminus was located at or near the center of the following repeat, yielding an estimated average size of 50-70 nt (Tang et al. 2002, 2005).

Northern blotting, using a probe against the repeat sequence of the clusters, revealed a series of discrete RNA products that were multiples of $\sim 68 \mathrm{nt}(68,136,204,272,340$ and $408 \mathrm{nt})$ for A. fulgidus (Tang et al. 2002) and of $\sim 60 \mathrm{nt}(60,180,360$ and $540 \mathrm{nt}$ ) for $S$. solfataricus (Tang et al. 2005). In both studies, the smallest RNA detected corresponded approximately to the estimated sizes of most of the cloned RNAs. The RNA size distribution is consistent with the processing of larger transcripts at regular spatial intervals and the sequencing data support, but do not establish, that processing occurs at or near the center of each repeat.

To gain further insight into the RNA products, transcription was examined from a small cluster of $S$. acidocaldarius (saci-4) (Chen et al. 2005) using a Northern blotting approach. Probes were prepared against complementary sequences of the terminal spacer sequence adjacent to the flanking sequence. Total RNA was extracted from exponentially growing and stationary phase cell cultures. The results revealed a series of bands that correspond to transcription from the direction of the flanking sequence (Figure 4). The pattern of larger discrete bands is quite similar to that obtained earlier for $S$. solfataricus P1 (Tang et al. 2005). What is different, however, is that diffuse bands range in size from 52 to $35 \mathrm{nt}$ for the exponentially growing sample, and from 52 to $30 \mathrm{nt}$ for the stationary phase sample (Figure 4A). This suggests that progressive trimming of the smallest discrete band of $58 \mathrm{nt}$ has occurred, possibly by exonucleases. Clearly, the yields of these smaller products increase, and their average size decreases, on going from the ex- ponential to stationary growth phase. The largest band ( 420 nt) exceeds the total size of the cluster (maximum 260 bp), which is consistent with the transcript extending beyond the cluster limits. Evidence is also provided for larger transcripts being produced by the complementary DNA strand in stationary phase cells (Figure 4B).

The observed sizes of the larger transcripts obtained from different clusters are consistent with transcription being initiated in a leader sequence within the flanking sequence adjoining the first repeat (Figure 2). The majority of the flanking

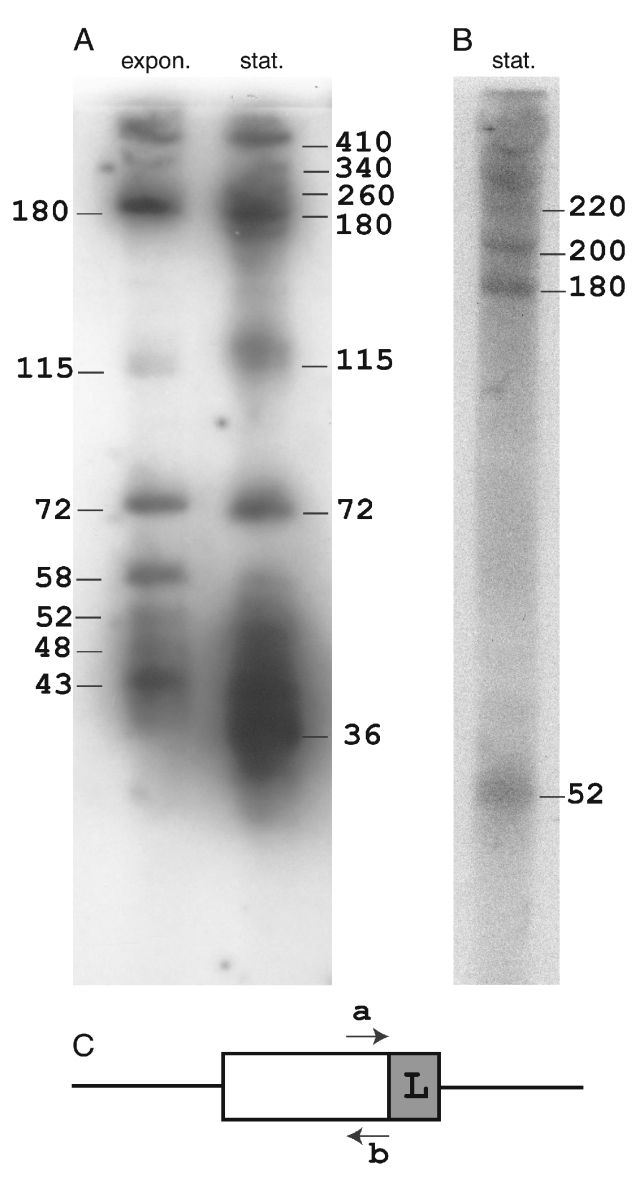

Figure 4. Northern blotting analyses of RNA transcripts obtained from the cluster saci-4 of S. acidocaldarius. (A) Transcripts from one strand were detected in total RNA extracted from cells grown to exponential (expon.) or stationary (stat.) phase. (B) Transcripts were observed from the complementary DNA strand at stationary phase. The approximate estimated nucleotide lengths of the RNA products are given. The minimal detection limit, using 26-nt. probes, was estimated at 15-16 nucleotides. (C) Denotes the location of the primers "a" and "b," and "L" indicates the location of the putative transcriptional leader within the flanking sequence. 
sequences (20 out of 26) carry a putative TATA-like motif immediately upstream from the first repeat which, if active, would produce an initation start at the center of this repeat. However, this property is also shared by repeat clusters lacking a flanking sequence and one of these, from the plasmid pKEF, produces transcripts (R.K. Lillestøl, unpublished data), suggesting that the flanking sequence is not primarily involved in transcriptional initiation.

\section{Derivation of the spacer sequences from extrachromosomal elements}

Recently, evidence has been presented for both archaea and bacteria that some spacer sequences correspond closely to sequences occurring in extrachromosomal elements some of which lie within ORFs. For the archaea, sequence similarities were found with Sulfolobus fuselloviruses and rudiviruses as well as with the conjugative plasmid pNOB8 (Mojica et al. 2005), whereas, for the bacteria, they were found for bacteriophages of Streptococcus and a prophage of Yersinia (Bolotin et al. 2005, Pourcel et al. 2005).

We tested all the archaeal spacer sequences for matches against the GenBank database and against our in-house archaeal genome database, which contains all the available ar- chaeal viral, plasmid and chromosomal sequences (Brügger et al. 2003). Several sequence matches were found, showing $88-100 \%$ identity with viruses, plasmids and chromosomes. They are listed in Table 6 and include those reported earlier (Mojica et al. 2005). Most matches were to ORFs; the few exceptions (Table 6) mainly correspond to predicted noncoding regions of chromosomes.

The majority of the positive archaeal matches are between spacer sequences of $S$. solfataricus and extrachromosomal elements of the related genera Sulfolobus and Acidianus. This strong bias probably reflects: (1) that Sulfolobus species are especially rich in repeat-spacer units (Table 1); and (2) that most sequenced archaeal viruses and plasmids derive from the related genera, Sulfolobus and Acidianus. Of the other positive matches, four were crenarchaeal (all to chromosomal sequences), eight were methanoarchaeal (mainly to phages or prophages), one was haloarchaeal and none were found to the haloarchaeal viruses, including SH1, His1 and His2 (Bamford et al. 2005, Bath et al. 2006) (Table 6).

The positions of the matching spacers for $S$. solfataricus are indicated in Figures $1 \mathrm{~B}$ and $1 \mathrm{C}$ and the identities and predicted functions of the matching ORFs are presented in Table 7. Most positive matches are to viruses, in particular to ORFs of the

Table 6. Spacer sequence matches to viruses, plasmids and chromosomes. Lengths of the sequence matches are given in columns 3-5 where sequence identity ranged from 88 to $100 \%$. For transposase genes only the best match is given. For M. thermoautotrophicus, there were three matches to phage $\psi \mathrm{M} 2$ ORF6 and two to prophage $\psi \mathrm{M} 100$ ORF31.

\begin{tabular}{|c|c|c|c|c|}
\hline Organism/plasmid & Spacer matches & Sense & Antisense & Noncoding regions \\
\hline P. aerophilum & P. aerophilum & & 39 & \\
\hline A. pernix & A. pernix & 32 & & \\
\hline \multirow[t]{10}{*}{ S. solfataricus } & ATV & $38,35,38,40,35,34,28$ & $39,39,37,39$ & \\
\hline & SIRV & $31,23,41$ & $40,31,29$ & \\
\hline & AFV6 & 29 & & \\
\hline & AFV7 & 25 & & \\
\hline & AFV8 & & 38 & \\
\hline & STIV & 27 & 23 & \\
\hline & pNOB8 & 25,38 & 40 & \\
\hline & A. fulgidus & & 32 & \\
\hline & S. solfataricus & & 40 & $40,35,38,32$ \\
\hline & S. tokodaii & & & 40,40 \\
\hline \multirow[t]{5}{*}{ S. tokodaii } & SIRV & 41 & & \\
\hline & SSV4 & 39 & & \\
\hline & pNOB8 & 38 & & \\
\hline & pKEF9 & 42 & & \\
\hline & S. tokodaii & 37 & & \\
\hline \multirow[t]{2}{*}{ S. acidocaldarius } & pKEF9 & 24 & & \\
\hline & S. acidocaldarius & & 35 & \\
\hline \multirow[t]{2}{*}{ pKEF9 } & SSV5 & & 27 & \\
\hline & SIRV & & 41 & \\
\hline \multirow[t]{4}{*}{ M. thermo-autotrophicus } & M. wolfeii & & & \\
\hline & prophage $\psi \mathrm{M} 100$ & 38 & 35,36 & 36 \\
\hline & M. marburgensis & & & \\
\hline & phage $\psi \mathrm{M} 2$ & $37,35,37$ & 36,36 & 38 \\
\hline M. acetivorans & M. acetivorans & & 38 & \\
\hline \multirow[t]{2}{*}{ M. mazei } & M. barkeri & & & \\
\hline & M. acetivorans & 36 & & \\
\hline N. pharaonis & N. pharaonis & & & 35,35 \\
\hline
\end{tabular}


Acidianus bicaudavirus ATV (62,730 bp) (Häring et al. 2005, Prangishvili et al. 2006). Matches also occurred to the similar rudiviruses SIRV1 and SIRV2 (Peng et al. 2001), the betalipothrixviruses AFV6, AFV7 and AFV8 (G. Vestergaard, University of Copenhagen, Denmark, unpublished data), the fusellovirus SSV4 (X. Peng, University of Copenhagen, Denmark, unpublished data) and the icosahedral virus STIV (Rice et al. 2004). Intriguingly, the cluster within the plasmid pKEF9 yielded matches with the rudivirus SIRV (Peng et al. 2001) and fusellovirus SSV5 (B. Greve, University of Copenhagen, Denmark, unpublished data; Table 7).

\section{A mechanism of defense}

Earlier evidence was provided for the presence of several RNAs in S. solfataricus cells that were antisense to transposase mRNAs, and it was inferred that these regulate the transpositional events of the numerous IS elements and MITEs present in the $S$. solfataricus chromosome, either by facilitating degradation of the transposase mRNAs, or by inhibiting their translation (Tang et al. 2005). However, sense fragments corresponding to transposase mRNA fragments were also present, which remain unexplained (Tang et al. 2005). Similarly, the spacer transcripts that match extrachromosomal ORF sequences also occur in both sense and antisense orientations, given that the clusters seem to be transcribed primarily in one direction (Tang et al. 2002, 2005; Figure 4). Thus, of the 29 ORF matches listed for $S$. solfataricus, 17 correspond to a mRNA and 12 are antisense (Table 7). This has led to some speculation as to whether the putative inhibitory mechanism acts at an RNA level (piRNA), as in the eukaryal interference RNA systems (siRNA and miRNA), or whether it occurs at the gene level with either sense or antisense transcripts annealing directly to a gene, thereby facilitating degradation of the viral genome (Marakova et al. 2006). These ideas are consistent with the presence of double-strand-specific ribonucleases in both crenarchaea and euryarchaea (Stolt et al. 1993, Ohtani et al. 2004) and the discovery of argonaute family proteins in the euryarchaea $P$. furiosus and A. fulgidus, which are an essential part of the RNA-induced silencing complex (RISC) in Eukarya (Parker et al. 2004, Song et al. 2004). The proposals are also reinforced by the prediction that some cas genes may have RNA-related polymerase or processing functions (Marakova et al. 2006).

\section{Conclusions}

Strong circumstantial evidence has been accrued over the past year for repeat clusters being involved in an antiviral cellular defense mechanism for almost all archaea and about 50\% of the bacteria investigated. The putative defence apparatus is shared by chromosomes and plasmids and is directed primarily against viruses. The genetic apparatus is complex and dynamic, undergoing rapid evolutionary change. It is likely to involve a large number of Cas proteins, including an essential core group, and some with more peripheral functions, which appear to be involved in the development of clusters and in the production and processing of the transcripts produced there-
Table 7. Spacer sequence matches between $S$. solfataricus strains P2 and $\mathrm{P} 1$ and viruses, plasmids and chromosomes. Matches of ATV and pIT3 to strain P1 are additional to those found for strain P2B, as illustrated in Figure 1. Symbol: * = an exclusive match to SIRV2. Literature references to the viral and plasmid sequences are given in the text for strain P2, and see Prato et al. 2006 regarding the pIT3 sequence.

\begin{tabular}{|c|c|c|c|}
\hline $\begin{array}{l}\text { Virus/plasmid/ } \\
\text { chromosome } \\
\text { matches }\end{array}$ & $\begin{array}{l}\text { Spacer } \\
\text { match } \\
\text { (mismatch) }\end{array}$ & Sense & Function \\
\hline \multicolumn{4}{|l|}{ S. solfataricus $P 2$} \\
\hline \multicolumn{4}{|l|}{ ATV } \\
\hline ORF61 & $35(1)$ & $\mathrm{S}$ & \\
\hline ORF127 & $38(0)$ & $\mathrm{S}$ & \\
\hline ORF145 & $39(0)$ & A & Virion protein \\
\hline ORF192 & $37(0)$ & A & ORF198, pING1 \\
\hline ORF326b & $39(1)$ & A & ParBc \\
\hline ORF529 & $34(0)$ & $\mathrm{S}$ & AAA-ATPase \\
\hline ORF545 & $35(2)$ & S & Membrane protein \\
\hline ORF618 & $40(0)$ & S & AAA-ATPase/virion protein \\
\hline ORF710 & $39(0)$ & A & \\
\hline ORF892 & $28(1)$ & $\mathrm{S}$ & VWA-domain protein \\
\hline ORF892 & $38(0)$ & $S$ & VWA-domain protein \\
\hline \multicolumn{4}{|l|}{ SIRVI/2 } \\
\hline ORF98 & $23(0)$ & $\mathrm{S}$ & \\
\hline ORF121* & $31(0)$ & A & Holliday junction resolvase \\
\hline ORF134 & $31(0)$ & S & Virion protein \\
\hline ORF268 & $41(5)$ & $S$ & \\
\hline ORF356 & $29(1)$ & A & Glycosyl transferase \\
\hline ORF510 & $40(2)$ & A & \\
\hline \multicolumn{4}{|l|}{ STIV } \\
\hline A109 & $27(0)$ & $\mathrm{S}$ & \\
\hline C557 & $23(0)$ & A & \\
\hline \multicolumn{4}{|l|}{$A F V s$} \\
\hline ORF267 AFV6 & $29(2)$ & $\mathrm{S}$ & \\
\hline ORF96 AFV7 & $25(1)$ & $\mathrm{S}$ & \\
\hline ORF593 AFV8 & $36(0)$ & A & AFV-type helicase \\
\hline \multicolumn{4}{|l|}{ pNOB8 } \\
\hline ORF315 & $40(3)$ & A & ParA \\
\hline ORF406 & $25(1)$ & $\mathrm{S}$ & IS element \\
\hline ORF1025 & $38(2)$ & $S$ & TrbE family \\
\hline \multicolumn{4}{|l|}{ Chromosome } \\
\hline $\begin{array}{l}\text { SSO1736 } \\
\text { (S. solfataricus) }\end{array}$ & $40(5)$ & A & IS element \\
\hline $\begin{array}{l}\text { AF1948 } \\
\quad(\text { A fulgidus })\end{array}$ & $32(3)$ & A & \\
\hline \multicolumn{4}{|l|}{ S. solfataricus $P 1$} \\
\hline ORF653 & $36(2)$ & $\mathrm{S}$ & Virion protein \\
\hline \multicolumn{4}{|l|}{ pIT3 } \\
\hline ORF80 & $35(0)$ & $\mathrm{S}$ & CopG \\
\hline
\end{tabular}

from. The clusters appear to be extended by DNA spacers derived, directly or indirectly, from the genes of invading viruses. Transcripts from the spacer sequences are thought to inhibit, or possibly regulate, viral propagation by hybridizing at an mRNA or gene level. 
Judging by its genetic complexity, this system must be important for survival of the archaeal cell in natural environments. This supposition is reinforced by the observation that large repeat clusters are present in all sequenced archaeal genomes, except that of Halobacterium sp. NRC-1 (Ng et al. 2000). Some insight into the latter exception may have been provided by a genomic mutation recently observed in $S$. solfataricus P2 (strain P2A in Redder and Garrett 2006). Strain P2 has been a laboratory strain for many years and, recently, a culture grown from a single colony was shown to exhibit a $124 \mathrm{kbp}$ deletion constituting $4 \%$ of the chromosome. The deletion included each of the four repeat clusters which carry a flanking sequence (ssol-103, ssol-95, ssol-32 and ssol-96 in Figure 1B) and all the cluster-related genes. Halobacterium $\mathrm{NRC} 1$ is, similarly, a common laboratory strain and it is likely that when these organisms are grown free from invading extra-chromosomal elements over a longer time period, there is a tendency to lose this complex and energy consuming genetic apparatus. This could also explain why many bacteria, particularly endosymbionts (Jansen et al. 2002), lack such a system.

\section{Acknowledgments}

We thank Jan Christiansen, Elfar Torarinsson, Qunxin She, Xu Peng and Gisle Vestergaard for helpful discussions and Hien Phan for help with DNA sequencing. The research was supported by grants from the Danish Research Council for Natural Science. Grants from Copenhagen University supported K. Brügger and P. Redder, with K. Brügger also receiving grants from the Danish Science Research Council.

\section{References}

Baliga, N.S., R. Bonneau, M.T. Facciotti et al. 2004. Genome sequence of Haloarcula marismortui: a halophilic archaeon from the Dead Sea. Genome Res. 14:2221-2234.

Bamford, D.H., J.J. Ravantti, G. Rönnholm, S. Laurinavicius, P. Kukkaro, M. Dyall-Smith, P. Somerharju, N. Kalkkinen and J.K.H. Bamford. 2005. Constituents of SH1, a novel lipid-containing virus infecting the halophilic archaeon Haloarcula hispanica. J. Virol. 79:9097-9107.

Bath, C., T. Cukalac, K. Porter and M.L. Dyall-Smith. 2006. His1 and His2 are distantly related, spindle-shaped haloviruses belonging to the novel virus group, Salterprovirus. Virology 350:228-239.

Bolotin, A., B. Quinquis, A. Sorokin and S.D. Ehrlich. 2005. Clustered regularly interspaced short palindrome repeats (CRISPRs) have spacers of extrachromosomal origin. Microbiology 151: 2551-2561

Brügger, K., P. Redder, Q. She, F. Confalonieri, Y. Zivanovic and R.A. Garrett. 2002. Mobile elements in archaeal genomes. FEMS Microbiol. Lett. 206:131-141

Brügger, K., P. Redder and M. Skovgaard. 2003. MUTAGEN: Multiuser tool for annotating genomes. Bioinformatics 19: 2480-2481.

Chen, L., K. Brügger, M. Skovgaard et al. 2005. The genome of Sulfolobus acidocaldarius, a model organism of the Crenarchaeota. J. Bacteriol. 187:4992-4999.

Falb, M., F. Pfeiffer, P. Palm, K. Rodewald, V. Hickmann, J. Tittor and D. Oesterhelt. 2005. Living with two extremes: conclusions from the genome sequence of Natronomonas pharaonis. Genome Res. 15:1336-1343.
Godde, J.S. and A. Bickerton. 2006. The repetitive DNA elements called CRISPRs and their associated genes: evidence of horizontal transfer among prokaryotes. J. Mol. Evol. 62:718-729.

Greve, B., S. Jensen, K. Brügger, W. Zillig and R.A. Garrett. 2004. Genomic comparison of archaeal conjugative plasmids from Sulfolobus. Archaea 1:231-239.

Haft, D.H., J. Selengut, E.F. Mongodin and K.E. Nelson. 2005. A guild of 45 CRISPR-associated (Cas) protein families and multiple CRISPR/Cas subtypes exist in prokaryotic genomes. PLoS Comput. Biol. 1:474-483.

Häring, M., G. Vestergaard, R. Rachel, L. Chen, R.A. Garrett and D. Prangishvili. 2005. Independent virus development outside a host. Nature 436:1101-1102.

Ishino, Y., H. Shinagawa, K. Makino, M. Amemura and A. Nakata. 1987. Nucleotide Sequence of the iap gene, responsible for alkaline phosphatase isozyme conversion in Escherichia coli, and identification of the gene product. J. Bacteriol. 169:5429-5433.

Jansen, R., J.D. Embden, W. Gaastra and L.M. Schouls. 2002. Identification of genes that are associated with DNA repeats in prokaryotes. Mol. Microbiol. 43:1565-1575.

Kawarabayasi, Y., Y. Hino, H. Horikawa et al. 2001. Complete genome sequence of an aerobic thermoacidophilic crenarchaeon Sulfolobus tokodaii strain 7. DNA Res. 8:123-140.

Lundgren, M., A. Andersson, L. Chen, P. Nilsson and R. Bernander. 2004. Three replication origins in Sulfolobus species: synchronous initiation of chromosome replication and asynchronous termination. Proc. Natl. Acad. Sci. USA 101:7046-7051.

Makarova, K.S., L. Aravind, N.V. Grishin, I.B. Rogozin and E.V. Koonin. 2002. A DNA repair system specific for thermophilic archaea and bacteria predicted by genomic context analysis. Nucleic Acids Res. 30:482-496.

Makarova, K.S., N.V. Grishin, S.A. Shabalina, Y.I. Wolf and E.V. Koonin. 2006. A putative RNA-interference-based immune system in prokaryotes: computational analysis of the predicted enzymatic machinery, functional analogies with eukaryotic RNAi, and hypothetical mechanisms of action. Biol. Direct 1. http://www.biology-direct.com/content/1/1/7/abstract.

Mojica, F.J., G. Juez and F. Rodriguez-Valera. 1993. Transcription at different salinities of Haloferax mediterranei sequences adjacent to partially modified PstI sites. Mol. Microbiol. 9:13-21.

Mojica, F.J., C. Ferrer, G. Juez and F. Rodriguez-Valera. 1995. Long stretches of short tandem repeats are present in the largest replicons of the archaea Haloferax mediterranei and Haloferax volcanii and could be involved in replicon partitioning. Mol. Microbiol. 17: $85-93$.

Mojica, F.J., C. Diez-Villasenor, J. Garcia-Martinez and E. Soria. 2005. Intervening sequences of regularly spaced prokaryotic repeats derive from foreign genetic elements. J. Mol. Evol. 60: 174-182.

Ng, W.V., S.P. Kennedy, G.G. Mahairas et al. 2000. Genome sequence of Halobacterium species NRC-1. Proc. Natl. Acad. Sci. USA. 97:12,176-12,181.

Ohtani, N., H. Yanagawa, M. Tomita and I. Mitsuhiro. 2004. Cleavage of double-stranded RNA by RNAse Hi from a thermoacidophilic archaeon, Sulfolobus tokodaii 7. Nucleic Acids Res. 32: 5809-5819.

Parker, J.S., S.M. Roe and D. Barford. 2004. Crystal structure of PIWI protein suggests mechanisms of siRNA recognition and slicer activity. EMBO J. 23:4727-4737.

Peng, X., H. Blum, Q. She, H. Domdey, S. Mallok, K. Brügger, R.A. Garrett, W. Zillig and D. Prangishvili. 2001. Sequences and replication of the archaeal rudiviruses SIRV1 and SIRV2: relationships to the archaeal lipothrixvirus SIFV and some eukaryal viruses. Virology 291:226-234. 
Peng, X., K. Brügger, B. Shen, L. Chen, Q. She and R.A. Garrett. 2003. Genus-specific protein binding to the large clusters of DNA repeats (Short Regularly Spaced Repeats) present in Sulfolobus genomes. J. Bacteriol. 185:2410-2417.

Pourcel, C., G. Salvignol and G. Vergnaud. 2005. CRISPR elements in Yersinia pestis acquire new repeats by preferential uptake of bacteriophage DNA, and provide additional tools for evolutionary studies. Microbiolgy 151:653-663.

Prangishvili, D., G. Vestergaard, M. Häring, R. Aramayo, T. Basta, R. Rachel and R.A. Garrett 2006. Structural and genomic properties of the hyperthermophilic archaeal virus ATV with an extracellular stage of the reproductive cycle. J. Mol. Biol. 359: 1203-1216.

Prato, S., R. Cannio, H.-P. Klenk, P. Contursi, M. Rossi and S. Bartolucci. 2006. pIT3, a cryptic plasmid isolated from the hyperthermophilic crenarchaeon Sulfolobus solfataricus IT3. Plasmid $56: 35-45$.

Redder, P. and R.A. Garrett. 2006. Mutations and rearrangements in the genome of Sulfolobus solfataricus P2. J. Bacteriol. 188: 4198-4206.

Rice, G., L. Tang, S. Stedman, F. Roberto, J. Spuhler, E. Gillitzer, J.E. Johnson, T. Douglas and M. Young. 2004. The structure of a thermophilic archaeal virus shows a double-stranded DNA viral capsid type that spans all domains of life. Proc. Natl. Acad. Sci. USA 101:7716-7720.

Sambrook, J. and D.W. Russell. 2001. Molecular cloning: a laboratory manual. 3rd Edn. Cold Spring Harbor Press, Woodbury, NY, 7.4-7.8.

Schleper, C., I. Holz, D. Janekovic, J. Murphy and W. Zillig. 1995. A multicopy plasmid of the extremely thermophilic archaeon Sulfolobus effects its transfer to recipients by mating. J. Bacteriol. 177:4417-4426.
She, Q., H. Phan, R.A. Garrett, S.V. Albers, K.M. Stedman and W. Zillig. 1998. Genetic profile of pNOB8 from Sulfolobus: the first conjugative plasmid from an archaeon. Extremophiles 2: 417-425.

She, Q., R.K. Singh, F. Confalonieri et al. 2001. The complete genome of the crenarchaeon Sulfolobus solfataricus P2. Proc. Natl. Acad. Sci. USA 98:7835-7840.

Song, J.J., S.K. Smith, G.J. Hannon and L. Joshua-Tor. 2004. Crystal structure of argonaute and its implications for slicer activity. Science 305:1434-1437.

Stolt, P. and W. Zillig. 1993. Structure specific ds/ss-RNase activity in the extreme halophile Halobacterium salinarium. Nucleic Acids Res. 21:5595-5599.

Tang, T.-H., J.-P. Bachellerie, T. Rozhdestvensky, M.-L. Bortolin, H. Huber, M. Drungowski, T. Elge, J. Brosius and A. Hüttenhofer. 2002. Identification of 86 candidates for small non-messenger RNAs from the archaeon Archaeoglobus fulgidus. Proc. Natl. Acad. Sci. USA 99:7536-7541.

Tang, T.-H., N. Polacek, M. Zywicki, H. Huber, K. Brügger, R.A. Garrett, J.P. Bachellerie and A. Hüttenhofer. 2005. Identification of novel non-coding RNAs as potential antisense regulators in the archaeon Sulfolobus solfataricus. Mol. Microbiol. 55: 469-481.

van Embden, J.D.A., T. van Gorkom, K. Kremer, R. Jansen, B.A.M. van der Zeijst and L.M. Schouls. 2000. Genetic variation and evolutionary origin of the direct repeat locus of Mycobacterium tuberculosis complex bacteria. J. Bacteriol. 182:2393-2401.

Zivanovic, Y.P., P. Lopez, H. Philippe and P. Forterre. 2002. Pyrococcus genome comparison evidences chromosome shufflingdriven evolution. Nucleic Acids Res. 30:1902-1910. 

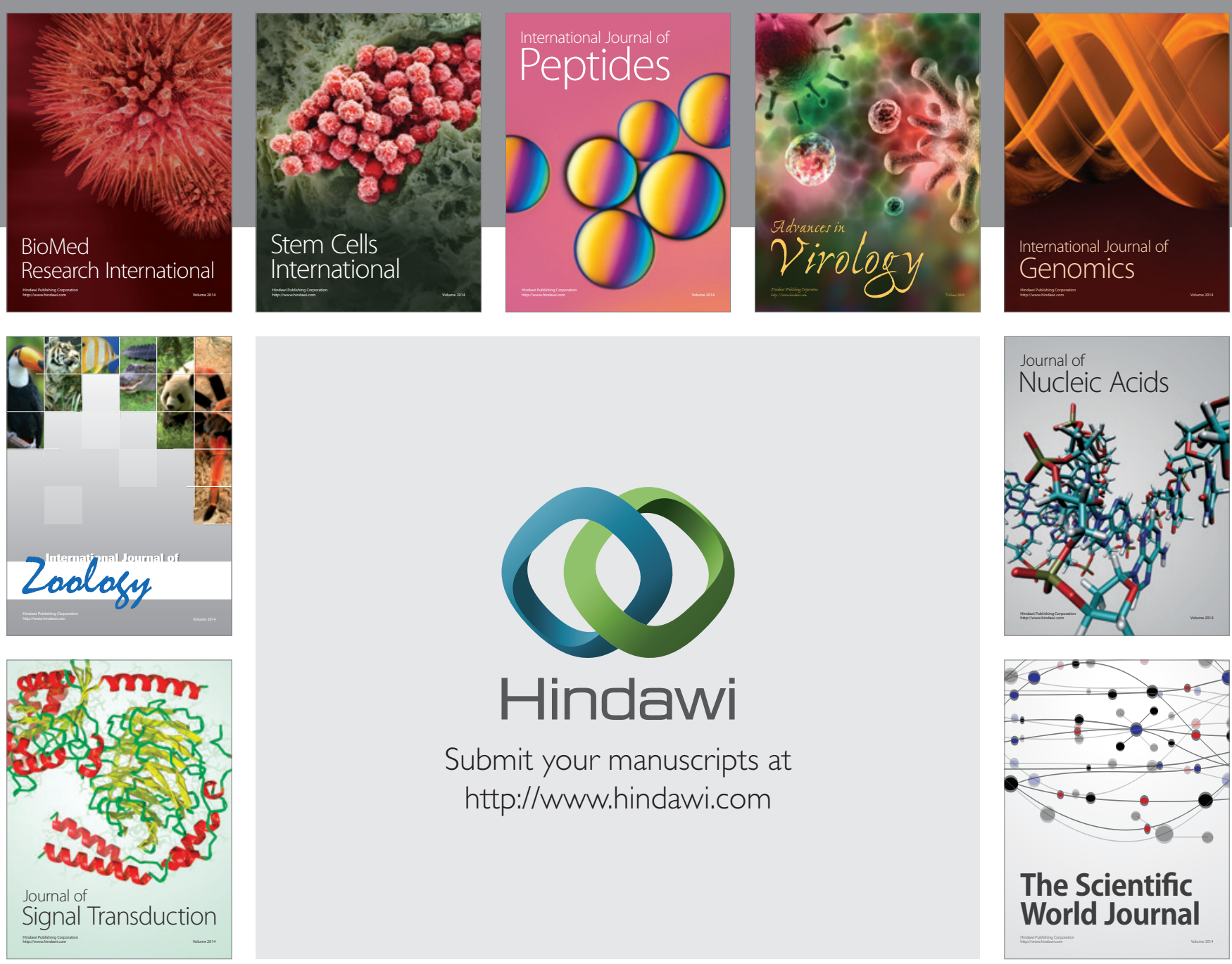

Submit your manuscripts at

http://www.hindawi.com
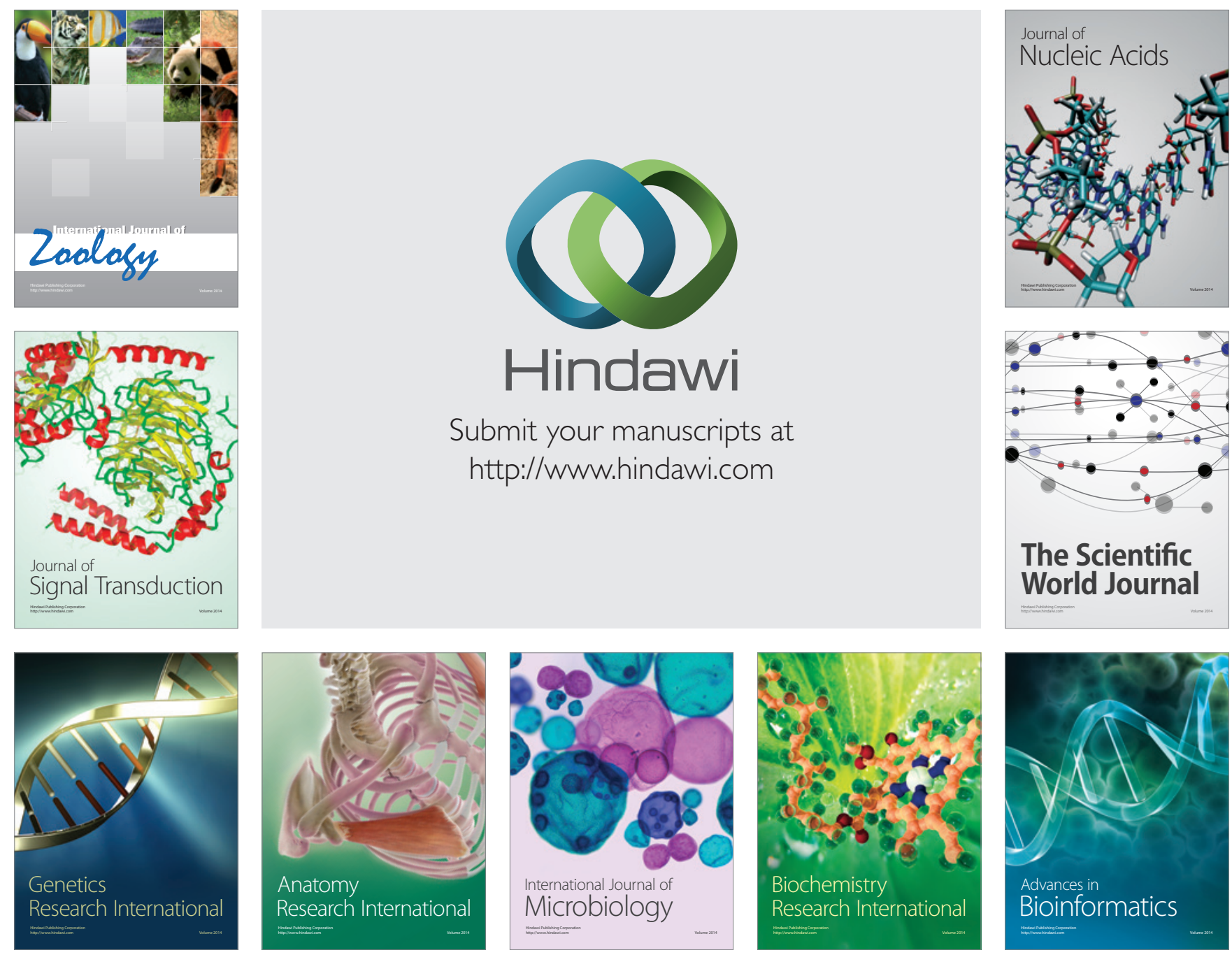

The Scientific World Journal
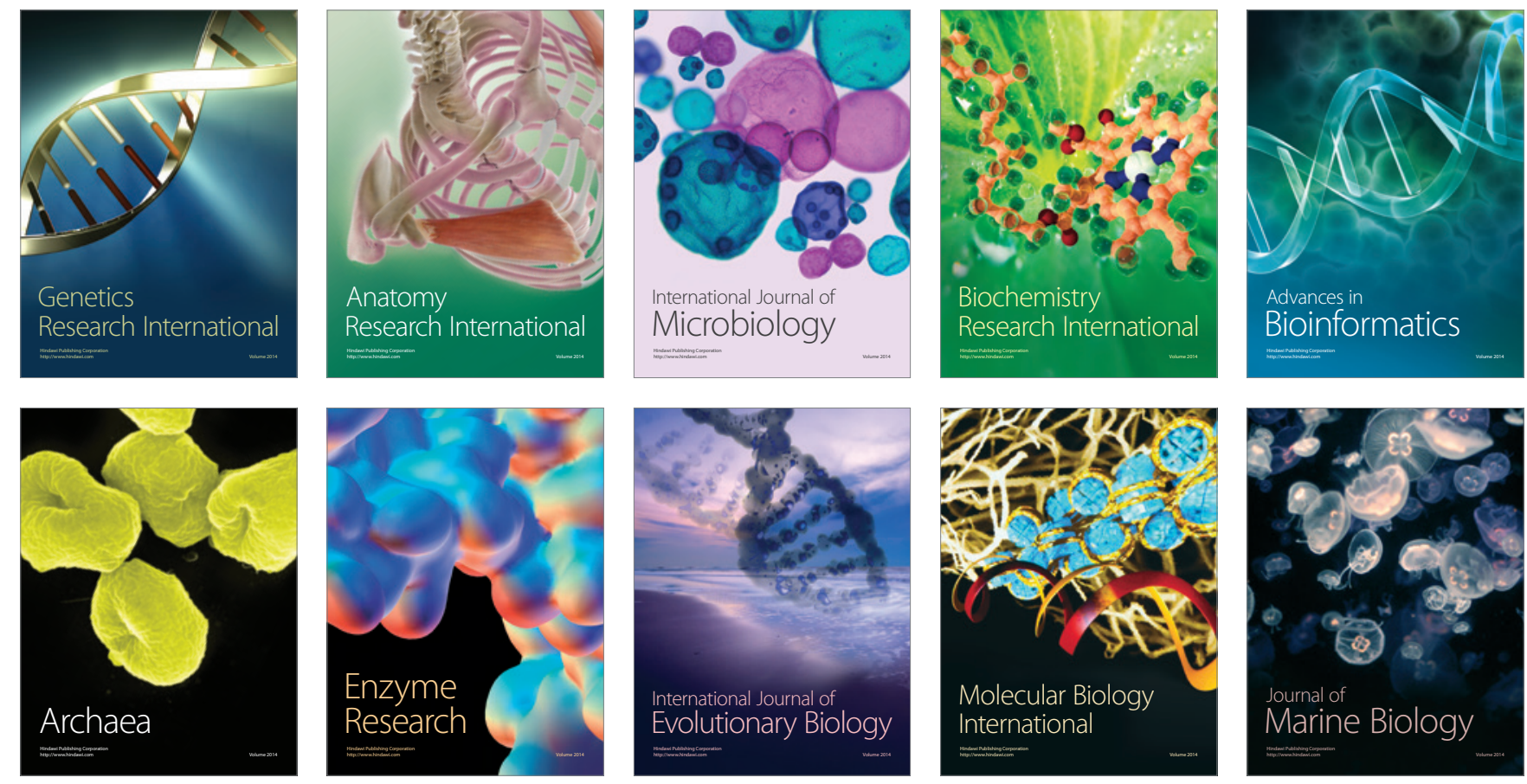